\title{
The utility and safety of linear endobronchial ultrasound-guided transbronchial needle aspiration (EBUS- TBNAl in the paediatric population
}

To the Editor:

Endobronchial ultrasound-guided transbronchial needle aspiration (EBUS-TBNA) in adults is an essential tool for investigating mediastinal and hilar lymphadenopathy. It is now integral to the diagnostic and staging algorithm for lung cancer [1], as well as the diagnosis of other malignancies, lymphoma and non-malignant granulomatous conditions, such as sarcoidosis and tuberculosis. The comparable diagnostic yield, along with decreased complications, has reduced the requirement for previously standard surgical biopsy sampling $[2,3]$.

There remains a paucity of data on convex probe (linear) EBUS in paediatric populations $[4,5]$ and no large European case series. The pathology in paediatric patients is distinct from adults due to the absence of lung cancer and logistical challenges, such as patient tolerance and scope diameter. We hypothesised that EBUS can be performed safely in the paediatric population and provide diagnostic material, obviating the need for more invasive procedures in most cases. The purpose of our study was to review the utility, safety and outcomes of paediatric EBUS in our centres.

A retrospective review of prospectively maintained bronchosopy and cytology databases was performed at two UK centres, Manchester Royal Infirmary and Guys and St Thomas's Trust, between May 2008 and November 2018. Cases aged $\leqslant 18$ years at time of procedure were included.

Procedures were performed by operators with experience of $\geqslant 200$ EBUS procedures in adults. EBUS-TBNA was performed using a standard EBUS bronchoscope (BF-UC180F-OL8, Olympus, Tokyo, Japan or EB-530US, Fujifilm, Japan). Lymph nodes were sampled using 22-guage needles employing a two-person technique, with a dedicated bronchoscopist but separate needle handler and labelled using the International Association for the Study of Lung Cancer lymph node map. Rapid on-site evaluation (ROSE) was used for all procedures, with consultant cytopathologist feedback on adequacy of lymph node sampling and preliminary diagnosis including presence of granuloma. All material was sent for microbiological analysis, including acid-fast bacilli (AFB).

Medical and anaesthetic records were reviewed for patient's demographics, ethnicity, indication for the procedure, final diagnosis, sedation, ventilation and any additional procedures or complications. Procedure, cytology and microbiology reports were reviewed to determine nodes sampled, nodal size, number of passes, immediate complications, as well as sample adequacy, cytology diagnosis, AFB stains and tuberculosis (TB) culture. Cases were considered to have a final diagnosis of TB if either culture positive or "clinically confirmed TB" if culture negative with granuloma on cytology and clinical response to TB treatment. Major complications were defined as death, bleeding requiring transfusion, advanced intervention or vasopressors, need for reversal agents, pneumothorax, mediastinitis or need for level 2-3 care.

All cases undergoing the procedure under general anaesthesia utilised a laryngeal mask airway size 4 or 5 performed with paediatric anaesthetists in paediatric theatres. Conscious sedation cases used a combination of opioid and benzodiazepines.

@ERSpublications

This first European case series demonstrates that EBUS-TBNA, well established in the diagnosis of mediastinal and hilar adenopathy in adults, is a safe and useful diagnostic alternative to invasive surgical biopsy in the paediatric population http://bit.ly/389Uvq4

Cite this article as: Al-Najjar H, Breen R, Santis G, et al. The utility and safety of linear endobronchial ultrasound-guided transbronchial needle aspiration (EBUS-TBNA) in the paediatric population. Eur Respir J 2020; 55: 1902277 [https://doi.org/10.1183/13993003.02277-2019]. 
40 patients aged $\leqslant 18$ years undergoing EBUS-TBNA for mediastinal or hilar abnormalities were identified. Basic demographics of the patients are displayed in table 1 . The indication in $85 \%(n=34)$ of procedures was isolated mediastinal or hilar adenopathy with a strong suspicion of new or recurrent tuberculosis, with the remaining $15 \%(n=6)$ for suspected lymphoma. The procedure was performed under general anaesthesia in $30 \%(n=12)$, all in Royal Manchester Children's Hospital, with the remainder under conscious sedation. There were no deaths or major complications (as defined by British Thoracic Society bronchoscopy guidelines criteria [6]) related to the procedures.

In total 70 nodal stations were sampled. Table 1 includes lymph node stations sampled. The final diagnosis breakdown in the 40 patients was TB (either cultured or clinically confirmed) in $67.5 \%(\mathrm{n}=27)$, reactive lymphadenopathy in $17.5 \%(\mathrm{n}=7)$, exclusion of TB recurrence in $7.5 \%(\mathrm{n}=3)$, haematological malignancy in $5 \%(n=2)$ and neurofibroma with granulomatous reaction in $2.5 \%(n=1)$. Of those initially suspected of having TB, the final diagnosis was TB in $76.5 \%(n=26)$; however, of those with suspected lymphoma the final diagnosis was lymphoma in $33 \%(n=2)$, TB in $17 \%(n=1)$ and reactive lymphadenopathy in $50 \%(n=3)$

The cytology displayed granuloma in $15 \%(n=6)$, necrotic granuloma in $52.5 \%(n=21)$ and no granuloma in $32.5 \%(n=13)$. Overall, $63 \%(n=17)$ of TB cases were culture positive (all fully sensitive), with the

\section{TABLE 1 Demographics and baseline characteristics (total $n=40$ )}

\begin{tabular}{|c|c|}
\hline \multicolumn{2}{|l|}{ Gender } \\
\hline Male & $20(50.0 \%)$ \\
\hline Female & $20(50.0 \%)$ \\
\hline Age years & $17(15-18)(12-18)$ \\
\hline \multicolumn{2}{|l|}{ Age } \\
\hline 12 years & $3(7.5 \%)$ \\
\hline 13 years & $2(5.0 \%)$ \\
\hline 14 years & $3(7.5 \%)$ \\
\hline 15 years & $6(15.0 \%)$ \\
\hline 16 years & $5(12.5 \%)$ \\
\hline 17 years & $10(25.0 \%)$ \\
\hline 18 years & $11(27.5 \%)$ \\
\hline \multicolumn{2}{|l|}{ Ethnicity } \\
\hline Asian & $15(37.5 \%)$ \\
\hline Caucasian & $8(20.0 \%)$ \\
\hline Black African & $12(30.0 \%)$ \\
\hline Black Caribbean & $4(10.0 \%)$ \\
\hline Arab & $1(2.5 \%)$ \\
\hline \multicolumn{2}{|l|}{ Initial indication } \\
\hline TB suspected & $32(80.0 \%)$ \\
\hline TB recurrence suspected & $2(5.0 \%)$ \\
\hline Lymphoma suspected & $6(15.0 \%)$ \\
\hline \multicolumn{2}{|l|}{ Number of nodes } \\
\hline 1 & $16(40.0 \%)$ \\
\hline 2 & $19(47.5 \%)$ \\
\hline 3 & $4(10.0 \%)$ \\
\hline 4 & $1(2.5 \%)$ \\
\hline \multicolumn{2}{|l|}{ Nodes $\#$} \\
\hline $10 \mathrm{~L}$ & $1 / 70(1.4 \%)$ \\
\hline $10 R$ & $6 / 70(8.6 \%)$ \\
\hline $11 \mathrm{~L}$ & $5(7.1 \%)$ \\
\hline $11 R$ & $3(4.3 \%)$ \\
\hline $12 R$ & $1(1.4 \%)$ \\
\hline $2 R$ & $2(2.9 \%)$ \\
\hline $4 \mathrm{~L}$ & $2(2.9 \%)$ \\
\hline $4 \mathrm{R}$ & $25(35.7 \%)$ \\
\hline 7 & 25 (35.7\%) \\
\hline Node size $e^{\pi}$ & $1.38(1.00-2.08)(0.55-3.50)$ \\
\hline
\end{tabular}


remaining cases negative. Culture-positive TB cases were more likely to display necrosis with granuloma on cytology than granuloma alone ( $82 \%$ versus $67 \%)$ and the nodes also tended to be larger in size $(2.05$ versus $1.72 \mathrm{~cm} ; \mathrm{p}=0.23)$ but neither reached statistical significance. Cytology had a sensitivity of $96 \%(95 \%$ CI $81-100 \%$ ) for TB in our cases and a negative predictive value of $88 \%$ (95\% CI $47-100 \%$ ). Culture compared with cytology had a positive predictive value of $63 \%$ (CI $42-81 \%$ ). $37 \%$ of culture-negative cases were identified on the basis of cytology alone and treated successfully. None of our cases required further surgical mediastinal sampling to ascertain the diagnosis.

Ours is the first exclusively European multicentre series reporting on linear EBUS safety and efficacy in the paediatric population. We encountered no major complications with an adequacy rate of $100 \%$ (assisted by ROSE). Our diagnostic yield is similar to previously reported studies in adult populations for both TB and lymphoma [7, 8]. Final diagnosis was changed from initial clinical suspicion by sampling in $23.5 \%$ of those suspected of having TB and $67 \%$ of those with suspected lymphoma. Whilst all our cases cultured fully sensitive $\mathrm{TB}$, our latest national figures showed $1.2 \%$ multidrug resistant $\mathrm{TB}$ and $11.4 \%$ with resistance to any first-line drug [9].

We identified two large $(n>20)$ multicentre studies in the literature. GILBERT et al. [10] reported a multicentre North American study on 21 paediatric patients undergoing EBUS-TBNA showing 95\% adequacy with $48 \%$ of cases providing diagnostic material and obviating the need for invasive surgical biopsy in $62 \%$. Like our study, they reported no major complications. In India, DHOoRIA et al. [11] included 55 paediatric EBUS-TBNA patients, most with suspected TB. They reported $92 \%$ adequacy with diagnostic material in $57 \%$, no major complications and $78 \%$ positive culture rate.

We believe the results are encouraging and may herald the wider use of EBUS-TBNA at tertiary centres in the paediatric population, hence avoiding invasive surgical procedures such as mediastinoscopy with their inherent risks. Both our centres are high-volume tertiary referral centres for EBUS. A limitation to the availability of paediatric EBUS is the smaller numbers of patients involved, which may make it difficult for paediatric pulmonologists to retain skills required to practice. Procedures at our institutes were performed by experienced adult pulmonologists liaising with paediatric colleagues.

Another issue for younger children requiring general anaesthetic is scope and airway size. EBUS scopes have an external diameter of 6.7 to $6.9 \mathrm{~mm}$, larger than paediatric bronchoscopes (2 to $4 \mathrm{~mm}$ ) and the tracheal diameter of younger children [12]. Adequate ventilation around the scope is needed as well as the ability to manoeuvre the EBUS scope.

Being retrospective, all shortcomings and limitations associated with retrospective studies are particular to ours, although both centres collected data prospectively. Also, both centres are tertiary paediatric centres with cytopathologists performing ROSE and high-volume EBUS through-put, making it difficult to extrapolate safety and efficacy figures widely. Future pathways may task certain centres with providing this day case, less invasive alternative to mediastinoscopy or thoracotomy, thus providing cost benefit as well as a safer risk profile [13].

In conclusion, our multicentre study shows EBUS-TBNA is safe and technically feasible in older children, when utilising an experienced paediatric/adult pulmonology and anaesthetic team. Furthermore, in the presence of ROSE performed by a cytopathologist, the adequacy rates and diagnostic yield are comparable to invasive surgical procedures with less risk.

Haider Al-Najjar @ ${ }^{1}$, Ronan Breen ${ }^{2}$, George Santis ${ }^{2}$ and Omendra Narayan ${ }^{3}$

${ }^{1}$ Respiratory Dept, Manchester University NHS Foundation Trust, Manchester, UK. ${ }^{2}$ Thoracic Medicine Dept, Guy's and St Thomas' NHS Foundation Trust, London, UK. ${ }^{3}$ Paediatric Respiratory Dept, Royal Manchester Children's Hospital, Manchester, UK.

Correspondence: Haider Al-Najjar, Respiratory Dept, Manchester University NHS Foundation Trust, Oxford Road, Manchester M13 9WL, UK. E-mail: Haider.al-najjar@mft.nhs.uk

Received: 25 Nov 2019 | Accepted after revision: 7 Jan 2020

Conflict of interest: None declared.

\section{References}

1 NICE. Lung Cancer Guidelines. 2019. http://guidance.nice.org.uk/ng122. Date last accessed: 14 October, 2019.

2 Navani N, Lawrence DR, Kolvekar S, et al. Endobronchial ultrasound-guided transbronchial needle aspiration prevents mediastinoscopies in the diagnosis of isolated mediastinal lymphadenopathy: a prospective trial. Am J Respir Crit Care Med 2012; 186: 255-260.

3 Annema JT, van Meerbeeck JP, Rintoul RC, et al. Mediastinoscopy vs endosonography for mediastinal nodal staging of lung cancer: a randomized trial. JAMA 2010; 304: 2245-2252.

4 Steinfort DP, Wurzel D, Irving LB, et al. Endobronchial ultrasound in pediatric pulmonology. Pediatr Pulmonol 2009; 44: 303-308. 
5 Goussard P, Gie R, Kling S, et al. The diagnostic value and safety of transbronchial needle aspiration biopsy in children with mediastinal lymphadenopathy. Pediatr Pulmonol 2010; 45: 1173-1179.

6 Du Rand IA, Blaikley J, Booton R, et al. British Thoracic Society guideline for diagnostic flexible bronchoscopy in adults: accredited by NICE. Thorax 2013; 68: i1-i44.

7 Moonim MT, Breen R, Fields PA, et al. Diagnosis and subtyping of de novo and relapsed mediastinal lymphomas by endobronchial ultrasound needle aspiration. Am J Respir Crit Care Med 2013; 188: 1216-1223.

8 Navani N, Molyneaux PL, Breen RA, et al. Utility of endobronchial ultrasound-guided transbronchial needle aspiration in patients with tuberculous intrathoracic lymphadenopathy: a multicentre study. Thorax 2011; 66: 889-893.

9 Public Health England. Tuberculosis in England Report. 2019. https://assets.publishing.service.gov.uk/government/ uploads/system/uploads/attachment_data/file/821334/Tuberculosis_in_England-annual_report_2019.pdf. Date last accessed: 19 December, 2019.

10 Gilbert CR, Chen A, Akulian JA, et al. The use of convex probe endobronchial ultrasound-guided transbronchial needle aspiration in a pediatric population: a multicenter study. Pediatr Pulmonol 2014; 49: 807-815.

11 Dhooria S, Madan K, Pattabhiraman V, et al. A multicenter study on the utility and safety of EBUS-TBNA and EUS-B-FNA in children. Pediatr Pulmonol 2016; 51: 1031-1039.

12 Masters IB, Ware RS, Zimmerman PV, et al. Airway sizes and proportions in children quantified by a video-bronchoscopic technique. BMC Pulmon Med 2006; 6: 5.

13 Sharples LD, Jackson C, Wheaton E, et al. Clinical effectiveness and cost-effectiveness of endobronchial and endoscopic ultrasound relative to surgical staging in potentially resectable lung cancer: results from the ASTER randomised control trial. Health Technol Assess 2012; 16: 1-75. 